Pacific Journal of Mathematics

FREE INTERPOLATION FOR HOLOMORPHIC FUNCTIONS 


\title{
FREE INTERPOLATION FOR HOLOMORPHIC FUNCTIONS REGULAR TO THE BOUNDARY
}

\section{JOAQUIM BRUNA AND FRANCESC TUGORES}

\begin{abstract}
We consider the class Lip $s$ of holomorphic functions in the unit disc satisfying a Lipschitz condition of order $s, 0<s \leq 1$ and the class $A^{1}$ of holomorphic functions $f$ such that the derivatives $f^{\prime}$ belong to the disc algebra. In this paper we give the complete characterization of the interpolation sets for these classes, thus completing the previous works of J. Bruna, E. M. Dyn'kin and E. P. Kronstadt.
\end{abstract}

I. Introduction and statement of results. This paper deals with interpolation problems in classes of holomorphic functions in the unit disc $D$ of the complex plane that have some regularity up to the boundary $T$. We denote by $A$ the disc algebra. We will consider the following classes included in $A$

$$
\begin{gathered}
\operatorname{Lip} s=\left\{f \in A ;|f(z)-f(w)| \leq \mathrm{const}|z-w|^{s}, z, w \in D\right\}, \\
A^{1}=\left\{f \in A ; f^{\prime} \in A\right\} .
\end{gathered}
$$

We could consider as well more regular classes Lip $s$ for $s>1$ or $A^{p}$ with $p=2,3, \ldots$. Our results and methods also apply to them with minor changes. To simplify the development we confine ourselves to the cases indicated.

For a given closed set $E \subset \bar{D}$, we denote by $\operatorname{Lip}_{s}(E)$ the space of functions on $E$ satisfying a Lipschitz condition of order $s$. For $C^{1}(E)$ we choose the Whitney definition. That is, $C^{1}(E)$ consists of the 1-jets $\phi=\left(\phi, \phi_{z}, \phi_{\bar{z}}\right)$, where $\phi, \phi_{z}, \phi_{\bar{z}}$ are continuous functions on $E$ such that

$$
\left|\phi(w)-\phi(z)-\phi_{z}(z)(w-z)-\phi_{\bar{z}}(z)(\bar{w}-\bar{z})\right|=o(|w-z|)
$$

uniformly in $w, z \in E$. By the Whitney extension theorem ([7], pg. 5) one can think of $\phi$ being the jet induced by a function $\phi$ in $C^{1}(C)$ with compact support (and then $\phi_{z}=\partial \phi / \partial z, \phi_{\bar{z}}=\partial \phi / \partial \bar{z}$ on $E$ ).

Definitions. $E$ is called an interpolation set for Lip $s$ if given any $\phi \in \operatorname{Lip}_{s}(E)$ there exists $f \in \operatorname{Lip} s$ such that $f=\phi$ on $E$. Similarly, we say that $E$ is an interpolation set for $A^{1}$ if given $\phi \in C^{1}(E)$ with $\phi_{\bar{z}}=0$ 
there exists $f \in A^{1}$ such that $f=\phi, f^{\prime}=\phi_{z}$ on $E$ (that is, for $A^{1}$ we require as well the interpolation of the derivative).

It is immediate that an interpolation set for both classes is a zero set (see [6]) and so $E \cap D$ must be a sequence $\left(a_{n}\right)$ such that

$$
\sum_{n}\left(1-\left|a_{n}\right|\right)<\infty
$$

and $E \cap T$ is a Carleson set (see [2]). From now on, all closed sets $E$ will be assumed to be of this type, and we write $E_{1}=E \cap D, E_{0}=E \cap T$.

In [4] the following theorem is proved:

TheOREM (Dyn'kin). $E \subset \bar{D}$ is an interpolation set for Lip $s, 0<s<1$ if and only if:

(a) $E_{1}$ satisfies the Carleson condition

$$
\inf _{n} \prod_{m \neq n} \frac{\left|a_{n}-a_{m}\right|}{\left|1-\bar{a}_{n} a_{m}\right|} \geq \delta>0
$$

(b) The distance function $\rho(z, E)=\inf _{w \in E}|z-w|$ satisfies the condition

$$
\text { “ } \sup _{z \in I} \rho(z, E) \geq \mathrm{const}|I| \text { for all intervals } I \subset T "
$$

where $|I|$ stands for the length of $I$.

From now on we shall refer to sets satisfying conditions (C) and (K) as (CK)-sets.

Dyn'kin's method does not apply to Lip 1 and $A^{1}$. Concerning $A^{1}$ it is proved in [1] using a different method that the boundary interpolation sets for $A^{1}$ (i.e. $E \subset T$ ) are also characterized by the condition $(\mathrm{K})$. In the case of Lip 1, Kronstadt proved in [6] that if $E$ is an interpolation set, then $E_{1}$ must be the union of two Carleson sequences. He found as well some sufficient conditions in special cases which allowed him to give an example of an interpolating sequence not satisfying (C). Thus, the characterization of the interpolation sets or Lip 1 must be different from that in Dyn'kin's theorem.

In this paper, we first obtain the characterization of the interpolation sets for Lip 1 and $A^{1}$, thus completing the previous works [1], [4] and [6]:

THEOREM 1. A closed set $E \subset \bar{D}$ is an interpolation set for Lip 1 if and only if the condition $(\mathrm{K})$ holds and $E_{1}$ is the union of two Carleson sequences. 
THEOREM 2. A closed set $E \subset \bar{D}$ is an interpolation set for $A^{1}$ if and only if it is $a(\mathrm{CK})$-set.

Our method consists essentially in applying the procedure of [1] twice. We note (see Remark 4.3.) that the first step can be adapted to obtain another proof of Dyn'kin's theorem, so that the paper also contains the characterization in the Lip $s$-case, $s<1$. We remark as well that the crucial condition (K) first appeared in the article [5] of A. M. Kotocigov and that we use metric properties of (CK)-sets obtained by Dyn'kin in [4].

The difference between $\operatorname{Lip} 1$ and $A^{1}$ is due to the fact that the interpolation of the derivative is not required for Lip 1. Note that $f \in \operatorname{Lip} 1$ is equivalent to $f^{\prime} \in H^{\infty}$. So, it is natural to consider the following definition:

DEFINITION. $E$ is called an interpolation set for Lip 1 in the strong sense if given $\phi \in \operatorname{Lip}_{1}(E)$ and $\left(\lambda_{n}\right) \in l^{\infty}$, there exists $f \in \operatorname{Lip} 1$ such that $f=\phi$ on $E$ and $f^{\prime}\left(a_{n}\right)=\lambda_{n}$ (here $\left.E_{1}=\left(a_{n}\right)\right)$.

Our last theorem illustrates this point:

TheOREM 3. A closed set $E \subset \bar{D}$ is an interpolation set for Lip 1 in the strong sense if and only if it is a (CK)-set.

In §II we have collected the properties of $(\mathrm{CK})$-sets used in the proofs as well as other auxiliary results. Section III is devoted to explaining the standard method used to construct the interpolating functions. In $§ I V$, we give the proof of Theorem 1. This section contains a constructive proof of the Carleson $H^{\infty}$-interpolation theorem for $(\mathrm{CK})$-sets which gives control on the derivative of the interpolating function (Lemma 4.2). Finally, Theorems 2 and 3 are proved in $\S \mathrm{V}$. In fact, the proof of Theorem 3 is quite similar to that of Theorem 2 and it is just sketched at the end.

II. Properties of (CK)-sets. Auxiliary results. First we introduce some general notations. As above, $\rho(z, E)$ denotes the Euclidean distance between $z$ and $E$ and $\rho\left(z, E_{0}\right)$ will denote the Euclidean distance between $z$ and $E_{0}$. The disc with center $z$ and radius $\varepsilon$ will be written $D(z, \varepsilon)$. We denote by $\psi(z, w)$ the pseudo-hyperbolic distance, i.e.,

$$
\psi(z, w)=\frac{|z-w|}{|1-\bar{z} w|}, \quad z, w \in D
$$


and we put $\psi\left(z, E_{1}\right)=\inf _{n} \psi\left(z, a_{n}\right)$. The pseudo-hyperbolic disc with center $z$ and radius $\varepsilon$ is written $N(z, \varepsilon)$. For an interval $I,|I|$ denotes the length of $I$ and

$$
R(I)=\{z \in D ; z /|z| \in I \text { and } 1-|I| / 2 \pi \leq|z| \leq 1\} .
$$

As usual, the notation $x \sim y$ will mean that the two variables $x, y$ satisfy $m<x / y<M$ for some constants $m, M>0$.

Obviously, condition (C) implies

$$
\psi\left(a_{n}, a_{m}\right) \geq \delta, \quad n \neq m
$$

that is, the points $\left(a_{n}\right)$ are uniformly separated in the pseudo-hyperbolic metric. Conversely, it is well known (see [10]) that (R) and the condition

$$
\sum_{a_{n} \in R(I)}\left(1-\left|a_{n}\right|\right) \leq \mathrm{const}|I|
$$

(that is, the measure with mass $1-\left|a_{n}\right|$ at $a_{n}$ is a Carleson measure) together imply $(\mathrm{C})$.

An easy computation shows that $\psi(z, w) \sim|z-w| /(1-|z|)$ if $\psi(z, w)<\min (|z|, 1 / 2)$, i.e., the pseudo-hyperbolic metric is roughly speaking $(1-|z|)^{-1}$ times the Euclidean metric. In particular for all $\delta>0$ sufficiently small one has

$$
D\left(a_{n}, c_{1} \delta\left(1-\left|a_{n}\right|\right)\right) \subset N\left(a_{n}, \delta\right) \subset D\left(a_{n}, c_{2} \delta\left(1-\left|a_{n}\right|\right)\right)
$$

for some positive absolute constants $c_{1}, c_{2}$. This means that condition (R) is equivalent to the existence of $\delta>0$ such that the discs $D_{n}=$ $D\left(a_{n}, \delta\left(1-\left|a_{n}\right|\right)\right)$ do not meet. Hence

$$
\psi\left(z, E_{1}\right) \sim 1, \quad z \notin \bigcup_{n} D_{n}, \quad \psi\left(z, E_{1}\right) \sim \frac{\left|z-a_{n}\right|}{1-\left|a_{n}\right|}, \quad z \in D_{n} .
$$

Replacing $\delta$ by $\delta / 2$, we can assume as well that

$$
|z-w| \geq \max \left(\delta\left(1-\left|a_{n}\right|\right), \delta\left(1-\left|a_{m}\right|\right)\right), \quad z \in D_{n}, w \in D_{m}, n \neq m .
$$

The following important concept and the lemma after it have been given by Dyn'kin in [4]:

DEFINITION. $E$ is called semi-Stolzian if for some $\lambda>1$

$$
\rho\left(a_{n}, E_{0}\right) \leq \lambda\left(1-\left|a_{n}\right|\right), \quad a_{n} \in E_{1} .
$$


Geometrically, this means that $E_{1}$ is contained in a "star" made up with Stolz sectors with vertex at the points at $E_{0}$ and constant amplitude.

LEMMA 2.1. If E satisfies condition (K), a countable number of points can be added to $E_{0}$ so that the resulting set $\tilde{E}$ is closed, semi-Stolzian and verifies $(\mathrm{K})$.

Hence, in proving the sufficiency of our three theorems, we may assume without loss of generality the sets $E$ are semi-Stolzian.

LEMMA 2.2. If $E$ is semi-Stolzian and satisfies condition ( $\mathrm{R})$, then

$$
\begin{gathered}
\rho\left(z, E_{0}\right) \sim \rho(z, E), \quad z \in D \backslash \bigcup_{n} D_{n}, \\
\rho\left(z, E_{0}\right) \sim 1-\left|a_{n}\right|, \quad \rho(z, E) \sim\left|z-a_{n}\right|, \quad z \in D_{n} .
\end{gathered}
$$

Proof. If $z \in D \backslash \cup_{n} D_{n}$ and $\rho(z, E)$ is attained at $a_{n} \in E_{1}$, one has

$$
\begin{aligned}
\rho\left(z, E_{0}\right) & \leq\left|z-a_{n}\right|+\rho\left(a_{n}, E_{0}\right) \leq\left|z-a_{n}\right|+\lambda\left(1-\left|a_{n}\right|\right) \\
& \leq\left(1+\lambda \delta^{-1}\right)\left|z-a_{n}\right|=\text { const } \rho(z, E) .
\end{aligned}
$$

If $z \in D_{n}$, then $\rho\left(z, E_{0}\right) \leq\left|z-a_{n}\right|+\rho\left(a_{n}, E_{0}\right) \leq(\delta+\lambda)\left(1-\left|a_{n}\right|\right)$. Also $\rho\left(z, E_{0}\right) \geq(1-|z|) \geq(1-\delta)\left(1-\left|a_{n}\right|\right)$. In particular $\rho\left(z, E_{0}\right) \geq$ $\delta^{-1}(1-\delta)\left|z-a_{n}\right|$. Since for $z \in D_{n} \rho\left(z, E_{1}\right)=\left|z-a_{n}\right|$ we find $\rho(z, E) \geq$ const $\left|z-a_{n}\right|$.

REMARK. The first relation in Lemma 2.2 is equivalent to the set $E$ being semi-Stolzian. We can now replace $E$ by $E_{0}$ in condition $(\mathrm{K})$. This is not always the case, as the following example shows. The set $E=$ $\left\{2^{n}\left(2^{n}+1\right)^{-1} e^{i / n}, n>1\right\} \cup\{1\}$ satisfies the condition (C) (it satisfies Newman's condition $1-\left|a_{n+1}\right| \leq \gamma\left(1-\left|a_{n}\right|\right), \gamma<1$ which implies (C), see [10]) and trivially condition (K) holds for $E_{0}$. But condition (K) fails for $I=\left\{e^{i t}, 0 \leq t \leq 1 / n\right\}$. Hence, (CK)-sets cannot be described in terms of $E_{1}$ and $E_{0}$ separately.

In conclusion we obtain the fundamental relation for the three distances involved for a semi-Stolzian (CK)-set

$$
\rho(z, E) \sim \rho\left(z, E_{0}\right) \psi\left(z, E_{1}\right), \quad z \in D .
$$

Throughout the paper this relation and the ones above will be systematically used without explicit mention. 
The following lemma is also proved in [4]:

LEMmA 2.3. If $E$ is a semi-Stolzian (CK)-set, there exists $\alpha, 0<\alpha<1$, such that for any $I \subset T$

$$
\begin{gathered}
\int_{R(I)} \rho\left(\zeta, E_{0}\right)^{-1-\alpha} d m(\zeta) \leq \mathrm{const}|I|^{1-\alpha} \\
\sum_{a_{n} \in R(I)}\left(1-\left|a_{n}\right|\right)^{1-\alpha} \leq \mathrm{const}|I|^{1-\alpha}
\end{gathered}
$$

Observe that (2) is the "discrete version" of (1). This lemma and the observation made at the beginning of this section show that conditions $(\mathrm{K})$ and $(\mathrm{R})$ taken together imply condition (C).

LEMMA 2.4. With the conditions stated above, one has

$$
\begin{aligned}
& \int_{D} \frac{\rho\left(\zeta, E_{0}\right)^{-1-\alpha}}{|\zeta-z|} d m(\zeta) \leq \mathrm{const} \rho\left(z, E_{0}\right)^{-\alpha}, \quad z \in D, \\
& \sum_{n} \frac{\left(1-\left|a_{n}\right|\right)^{1-\alpha}}{\left|z-a_{n}\right|} \leq \mathrm{const} \rho\left(z, E_{0}\right)^{-\alpha}, \quad z \notin \bigcup_{n} D_{n}, \\
& \sum_{m \neq n} \frac{\left(1-\left|a_{m}\right|\right)^{1-\alpha}}{\left|z-a_{m}\right|} \leq \mathrm{const} \rho\left(z, E_{0}\right)^{-\alpha}, \quad z \in D_{n} .
\end{aligned}
$$

Proof. (3) was obtained from (1) in [1]. The relations (4) and (5) will follow from (2) in the same manner. If $z \notin \cup_{n} D_{n}$, then $\left|z-a_{n}\right| \geq$ $\rho(z, E) \geq c \rho\left(z, E_{0}\right)$. Writing $P_{k}=\left\{a_{n}: c 2^{k} \rho\left(z, E_{0}\right) \leq\left|z-a_{n}\right| \leq\right.$ $\left.c 2^{k+1} \rho\left(z, E_{0}\right)\right\}$ the sum in (4) is

$$
\begin{aligned}
& \sum_{k=0}^{\infty} \sum_{a_{n} \in P_{k}} \frac{\left(1-\left|a_{n}\right|\right)^{1-\alpha}}{\left|z-a_{n}\right|} \\
& \quad \leq \operatorname{const} \rho\left(z, E_{0}\right)^{-1} \sum_{k=0}^{\infty} 2^{-k} \sum_{a_{n} \in P_{k}}\left(1-\left|a_{n}\right|\right)^{1-\alpha} .
\end{aligned}
$$

But $D \cap D\left(z, c 2^{k+1} \rho\left(z, E_{0}\right)\right)$, if not empty, is contained in a $R(I)$ with $|I|=c 2^{k+1} \rho\left(z, E_{0}\right)+1-|z| \leq$ const $2^{k} \rho\left(z, E_{0}\right)$. Then (4) follows from (2).

If $z \in D_{n}$, then $\left|z-a_{m}\right| \geq c \rho\left(z, E_{0}\right)$ for $m \neq n$ and (5) is obtained in the same way. 
LEMMA 2.5. If $s \leq 1$ and $\alpha$ is the same as in Lemma 2.4,

(6)

$$
\begin{aligned}
& \int_{D} \frac{\rho(\zeta, E)^{s-1}}{\rho\left(\zeta, E_{0}\right)^{s+\alpha} \psi\left(\zeta, E_{1}\right)} \frac{d m(\zeta)}{|\zeta-z|} \\
& \quad= \begin{cases}O\left(\rho\left(z, E_{0}\right)^{-\alpha} \psi\left(z, E_{1}\right)^{s-1}\right) & \text { if } s<1, \\
O\left(\rho\left(z, E_{0}\right)^{-\alpha}\left(\text { const }+\left|\log \psi\left(z, E_{1}\right)\right|\right)\right), & s=1 .\end{cases}
\end{aligned}
$$

Also, if $H(z)=\{\zeta:|\zeta| \leq 1,|\zeta-z| \leq \rho(z, E) / 2\}$, one has

$$
\begin{aligned}
& \int_{H(z)} \frac{\rho(\zeta, E)^{s-2}}{\rho\left(\zeta, E_{0}\right)^{s+\alpha} \psi\left(\zeta, E_{1}\right)} \frac{d m(\zeta)}{|\zeta-z|} \\
& \quad=O\left(\rho\left(z, E_{0}\right)^{-\alpha-1} \psi\left(z, E_{1}\right)^{s-2}\right), \quad s \leq 1 .
\end{aligned}
$$

Proof. The integrand in (6) is bounded by some constant times $\rho\left(\zeta, E_{0}\right)^{-1-\alpha} /|\zeta-z|$ for $\zeta$ in $D \backslash \cup_{n} D_{n}$. Hence, (3) shows that the integral over $D \backslash \cup_{n} D_{n}$ satisfies the required estimate. The integral over $\cup_{n} D_{n}$ is bounded by

$$
\sum_{n}\left(1-\left|a_{n}\right|\right)^{1-s-\alpha} \int_{D_{n}} \frac{\left|\zeta-a_{n}\right|^{s-2}}{|\zeta-z|} d m(\zeta) .
$$

Now, a careful computation shows that

$$
\int_{D_{n}} \frac{\left|\zeta-a_{n}\right|^{s-2}}{|\zeta-z|} d m(\zeta) \leq \begin{cases}\operatorname{const} \frac{\left(1-\left|a_{n}\right|\right)^{s}}{\left|z-a_{n}\right|} & \text { if } z \notin D_{n}, s \leq 1, \\ \operatorname{const}\left|z-a_{n}\right|^{s-1} & \text { if } z \in D_{n}, s<1, \\ \operatorname{const}\left|\log \frac{\left|z-a_{n}\right|}{1-\left|a_{n}\right|}\right| & \text { if } z \in D_{n}, s=1 .\end{cases}
$$

If $z \notin \cup_{n} D_{n}$, the integral over $\cup_{n} D_{n}$ is then bounded by some constant times the expression in (4) and so (6) is proved (recall that $\psi\left(z, E_{1}\right) \sim 1$ in $D \backslash \cup D_{n}$ ). If $z \in D_{n}$, the same holds for the integral over $\cup_{m \neq n} D_{m}$, this time using (5). Finally, the contribution of $D_{n}$ for $z \in D_{n}$ is bounded by

$$
\begin{array}{ll}
\operatorname{const}\left(1-\left|a_{n}\right|\right)^{1-s-\alpha}\left|z-a_{n}\right|^{s-1}, & s<1, \\
\operatorname{const}\left(1-\left|a_{n}\right|\right)^{-\alpha}\left|\log \psi\left(z, E_{1}\right)\right|, & s=1
\end{array}
$$

which are of the desired type. 
To obtain (7) it is enough to use the fact that $\rho(\zeta, E) \sim \rho(z, E)$, $\rho\left(\zeta, E_{0}\right) \sim \rho\left(z, E_{0}\right)$ for $\zeta$ in $H(z)$.

We denote by $B_{E}(z)$ the Blaschke product corresponding to $E_{1}$ :

$$
B_{E}(z)=\prod_{n} \frac{\left|a_{n}\right|}{a_{n}} \frac{z-a_{n}}{1-\bar{a}_{n} z}, \quad z \in D .
$$

As proved in [9], the condition (C) is equivalent to

$$
\left|B_{E}(z)\right| \sim \psi\left(z, E_{1}\right), \quad z \in D .
$$

Also it is shown in [4] that if $E$ is a semi-Stolzian (CK)-set, then

$$
\left|B_{E}^{(k)}(z)\right| \leq \text { const } \rho\left(z, E_{0}\right)^{-k}, \quad z \in D, k \geq 1 .
$$

Recall that $B_{E}$ is $C^{\infty}$ in $\bar{D} \backslash E_{0}$ and so the above estimate also holds for $z \in T \backslash E_{0}$.

The condition (K) implies (see [1], [4]) the existence, for each $r>0$, of an outer function $F_{r}$ which is $C^{\infty}$ in $T \backslash E_{0}$ and such that

$$
\begin{gathered}
\left|F_{r}(z)\right| \sim \rho\left(z, E_{0}\right)^{r}, \quad z \in \bar{D} \\
\left|F_{r}^{(k)}(z)\right| \leq \text { const } \rho\left(z, E_{0}\right)^{r-k}, \quad z \in \bar{D} \backslash E_{0}, k \geq 1 .
\end{gathered}
$$

Also, the above estimates on $B_{E}$ and $F_{r}$ will be systematically used without explicit mention.

For further reference we state a lemma proved in [1] that will be used in several estimates.

LemMA 2.6. Let $\varphi$ be of class $C^{n+1}$ in some arc $J=[a, b]$ of $T$, let $c=(a+b) / 2$ be the middle point of $J$ and let $A(z)$ be defined by

$$
A(z)=\int_{a}^{b} \frac{e^{i t}}{\left(e^{i t}-z\right)^{n+1}} \varphi\left(e^{i t}\right) d t, \quad z \in D .
$$

Put $\varphi^{0}=\varphi$ and

$$
\varphi^{(k)}\left(e^{i t}\right)=e^{-i t} \frac{d}{d t} \varphi^{(k-1)}\left(e^{i t}\right), \quad k=1, \ldots, n+1 .
$$

Then, for $z=r e^{i c}, 0 \leq r<1$,

$$
|A(z)| \leq \mathrm{const}\left(\sum_{k=0}^{n-1} \frac{M_{k}}{\left|z-e^{\imath a}\right|^{n-k}}+M_{n}+|J| M_{n+1}\right)
$$

where $M_{k}=\max \left\{\left|\varphi^{(k)}\left(e^{i t}\right)\right|, a \leq t \leq b\right\}$. 
Finally the following lemma clarifies the structure of the union of two Carleson sequences.

LEMMA 2.7. For a sequence $E_{1}=\left(a_{n}\right)$ in $D$ the following are equivalent:

(a) $E_{1}$ is the union of two Carleson sequences.

(b) For each $a_{n}$, there exists $a_{m}$ such that

$$
\prod_{k \neq n, m} \psi\left(a_{n}, a_{k}\right) \geq \delta>0 .
$$

(c) The sequence $\left(a_{n}\right)$ is either a Carleson sequence or it can be rearranged $E_{1}=\left(b_{n}\right) \cup\left(c_{n}\right)$, where $\left(b_{n}\right)$ and $\left(c_{n}\right)$ are Carleson sequences, $\psi\left(b_{n}, c_{n}\right)=\psi\left(b_{n}, E_{1} \backslash b_{n}\right)=\sigma_{n} \rightarrow 0$, and $\psi\left(b_{n}, a\right) \geq \delta$ if $a \neq c_{n}, b_{n}$.

Proof. (b) $\Rightarrow$ (c) is in fact proved in [6]. To see that (a) $\Rightarrow(b)$, suppose $E_{1}=F_{1} \cup G_{1}$ where $F_{1}=\left(b_{n}\right)$ and $G_{1}=\left(c_{n}\right)$ are Carleson sequences. Given $b_{n} \in F_{1}$ let $c \in G_{1}$ be such that $\psi\left(b_{n}, G_{1}\right)=\psi\left(b_{n}, c\right)$. Since

$$
\left|B_{G_{1}}\left(b_{n}\right)\right| \sim \psi\left(b_{n}, G_{1}\right),
$$

we have $\Pi_{c_{m} \neq c} \psi\left(b_{n}, c_{m}\right) \geq$ const. Also, $\Pi_{b_{m} \neq b_{n}} \psi\left(b_{n}, b_{m}\right) \geq$ const, because $F_{1}$ is a Carleson sequence and so (b) follows.

III. The standard method of interpolation in regular classes. We will systematically use the following procedure to obtain a holomorphic function $f$ in $D$ which interpolates a given function $\phi$ on the set $E$. In all cases (except in Lemma 4.2), $\phi$ is a continuous function in $\bar{D}$ which is $C^{\infty}$ outside $E$. If $h$ is in the disc algebra, is $C^{\infty}$ in $\bar{D} \backslash E$, vanishes only on $E$ and is such that $\bar{\partial} \phi / h$ is integrable on $D$, we define

$$
v(z)=\frac{-1}{\pi} \int_{D} \frac{\bar{\partial} \phi(\zeta)}{h(\zeta)} \frac{d m(\zeta)}{\zeta-z}, \quad z \in D \backslash E .
$$

We claim that $v(z)$ is a continuous function in $\bar{D} \backslash E$. This is because $|\zeta-z|^{-1}$ is integrable and $\bar{\partial} \phi / h$ is bounded near $z$ (Cauchy transform of a bounded function) and because near $E$ where $|\zeta-z|^{-1}$ is bounded, $\bar{\partial} \phi / h$ is integrable. Since $\bar{\partial} v=\bar{\partial} \phi / h$, the function

$$
f=\phi-v h
$$

is holomorphic in $D$, continuous in $\bar{D} \backslash E$.

The hypo-ellipticity of the $\bar{\partial}$-equation implies that $v$ is $C^{\infty}$ in $D \backslash E$. Now we wish to obtain a formula for $\partial v(z)$ for $z \in D \backslash E$ and to show that $\partial v$ has a continuous extension to $\bar{D} \backslash E$. Fix $z \in \bar{D} \backslash E$. For $|w|<1$, $|w-z|<\rho(z, E) / 4$ break the integral defining $v(w)$ into two parts corresponding to $D \backslash H(z)$ and $H(z)$, and apply Stoke's theorem to the 
contribution of $H(z)$. In the expression obtained one can differentiate under the integral sign because the resulting integrals are still convergent. Finally we obtain

$$
\begin{aligned}
& \partial v(w)= \frac{-1}{\pi} \int_{D \backslash H(z)} \frac{\bar{\partial} \phi(\zeta)}{h(\zeta)} \frac{d m(\zeta)}{(\zeta-w)^{2}}-\frac{1}{\pi} \int_{H(z)} \frac{\partial}{\partial \zeta}\left(\frac{\bar{\partial} \phi}{h}\right)(\zeta) \frac{d m(\zeta)}{\zeta-w} \\
&-\frac{1}{2 \pi i} \int_{\partial H(z)} \frac{\bar{\partial} \phi(\zeta)}{h(\zeta)} \frac{d \bar{\zeta}}{\zeta-w}, \\
&|w|<1,|w-z|<\rho(z, E) / 4
\end{aligned}
$$

Putting $w=z$ we obtain the desired formula for $\partial v(z), z \in D \backslash E$.

If $z \in T \backslash E$, all terms have continuous extensions to the $\operatorname{arc}\{w \in T$ : $|w-z|<\rho(z, E) / 4\}$. This is clear for the first and second terms by the same argument used above for $v(z)$. The fact that $\bar{\partial} \phi / h$ is $C^{\infty}$ outside $E$ assures the same for the third one. Thus $\partial v$ extends continuously to $T \backslash E$.

In particular, if $\phi$ is more regular, say $C^{1}$ in $\bar{D}$, then $f^{\prime}(z)=\partial \phi(z)-$ $\partial(v h)(z)$ also has a continuous extension to $T \backslash E$.

We shall refer to $f$ as the function obtained applying the standard method of interpolation to the pair $(\phi, h)$ and write $f=P(\phi, h)$.

\section{Proof of Theorem 1.}

Proof of necessity. Suppose that $E$ is an interpolation set for Lip 1. Then $E_{1}$ is an interpolation set for Lip 1 and so (see [6]) satisfies the condition (b) in Lemma 2.7.

In order to prove condition (K), we can assume by Lemma 2.7 , that $E_{1}$ is either a Carleson sequence or it can be rearranged as in (c) of that lemma.

It is enough to prove condition (K) for $|I|$ small enough. We shall show that if $|I|$ is sufficiently small, there exists $\zeta \in R(I)$ such that $\rho(\zeta, E) \geq c|I|$ and $\rho(\zeta, \partial R(I)) \geq c|I|$. If $\zeta_{0}$ is the center of $R(I)$ and $E_{1} \cap D\left(\zeta_{0},|I| / 8 \pi\right)=\varnothing$ just choose $\zeta=\zeta_{0}$. If $b_{n} \in E_{1} \cap D\left(\zeta_{0},|I| / 8 \pi\right)$ then $1-\left|b_{n}\right| \sim|I|$. So we can suppose that $|I|$ is so small that in case some $b_{n}$ exists in $E_{1} \cap D\left(\zeta_{0},|I| / 8 \pi\right)$ the corresponding $c_{n}$ is in $D\left(\zeta_{0},|I| / 6 \pi\right)$, say (this is only in case $E_{1}$ is not a Carleson sequence), and the other points of $E_{1}$ are outside $D\left(\zeta_{0},|I| / 4 \pi\right)$, say. Then just choose $\zeta$ such that $\left|\zeta-\zeta_{0}\right|=|I| / 5 \pi$. Once the existence of such $\zeta$ is proved the same argument as in [4] serves to prove condition $(\mathrm{K})$. 
Proof of sufficiency. Suppose that $E$ is a set for which condition (K) holds and $E_{1}$ is the union of two Carleson sequences. To see that $E$ is an interpolation set for Lip 1 we can assume by Lemma 2.1. that $E$ is a semi-Stolzian set. The first step deals with the case that $E_{1}$ is a Carleson sequence.

LEMMA 4.1. Every semi-Stolzian (CK)-set $E$ is an interpolation set for Lip 1 .

Proof of Lemma 4.1. By a theorem of Whitney's type (see [8], pg. 174) we can suppose that $\phi \in C^{\infty}(\mathbf{C} \backslash E), \phi \in \operatorname{Lip}_{1}(\mathbf{C})$ and that

$$
\begin{aligned}
|\bar{\partial} \phi(\zeta)| & \leq \text { const } ;|\partial \phi(\zeta)| \leq \text { const, } \quad \zeta \notin E, \\
|\partial \bar{\partial} \phi(\zeta)| & \leq \text { const } \rho(\zeta, E)^{-1}, \quad \zeta \notin E .
\end{aligned}
$$

Take $h=B_{E} F_{1+\alpha}$ where $\alpha$ is the same as in Lemmas 2.4 and 2.5 and consider $f=P(\phi, h)$. Suppose we prove:

$$
\begin{aligned}
&|v(z)|=O\left(\rho\left(z, E_{0}\right)^{-\alpha}\left(c+\left|\log \psi\left(z, E_{1}\right)\right|\right)\right), \quad z \in \bar{D} \backslash E, \\
&|\partial v(z)|=O\left(\rho\left(z, E_{0}\right)^{-\alpha-1} \psi\left(z, E_{1}\right)^{-1}\left(c+\left|\log \psi\left(z, E_{1}\right)\right|\right)\right), \\
& z \in \bar{D} \backslash E .
\end{aligned}
$$

Then, since

$$
|h(z)| \sim \rho\left(z, E_{0}\right)^{1+\alpha} \psi\left(z, E_{1}\right) \quad \text { and } \quad\left|h^{\prime}(z)\right| \leq \text { const } \rho\left(z, E_{0}\right)^{\alpha}
$$

it follows that

$$
\begin{gathered}
|v(z) h(z)|=O\left(\rho\left(z, E_{0}\right) \psi\left(z, E_{1}\right)\left(c+\left|\log \psi\left(z, E_{1}\right)\right|\right)\right), \quad z \in \bar{D} \backslash E, \\
|\partial(v h)(z)|=O\left(c+\left|\log \psi\left(z, E_{1}\right)\right|\right), \quad z \in \bar{D} \backslash E .
\end{gathered}
$$

The second implies that $f^{\prime}=\partial \phi-\partial(v h)$ is bounded in $D \backslash \cup_{n} D_{n}$ hence bounded in $D$ by the maximum modulus theorem. Therefore $f$ is in Lip 1 and now the first relation implies that $f$ interpolates $\phi$ on $E$. So to prove the Lemma it is enough to show that (8) and (9) hold (in fact observe that we just need (9) for $z \in D \backslash \cup_{n} D_{n}$ ). Here

$$
|v(z)| \leq \mathrm{const} \int_{D} \frac{1}{\rho\left(\zeta, E_{0}\right)^{1+\alpha} \psi\left(\zeta, E_{1}\right)} \frac{d m(\zeta)}{|\zeta-z|}
$$


and (8) follows from (6) in Lemma 2.5. For $\partial v(z), z \in D \backslash E$ we have

$$
\begin{aligned}
|\partial v(z)| \leq & \text { const } \int_{D \backslash H(z)} \frac{1}{\rho\left(\zeta, E_{0}\right)^{1+\alpha} \psi\left(\zeta, E_{1}\right)} \frac{d m(\zeta)}{|\zeta-z|^{2}} \\
& + \text { const } \int_{H(z)} \frac{\rho(\zeta, E)^{-1}}{\rho\left(\zeta, E_{0}\right)^{1+\alpha} \psi\left(\zeta, E_{1}\right)} \frac{d m(\zeta)}{|\zeta-z|} \\
& + \text { const }\left|\int_{\partial H(z)} \frac{\bar{\partial} \phi(\zeta)}{h(\zeta)} \frac{d \bar{\zeta}}{\zeta-z}\right| .
\end{aligned}
$$

In $D \backslash H(z),|\zeta-z| \geq \rho(z, E) / 2$ and (6) shows that the first term satisfies the estimate (9). So does the second one, by (7). Finally, let $\gamma_{1}=\{\zeta \in$ $\bar{D}:|\zeta-\mathrm{z}|=\rho(z, E) / 2\}$ and $\gamma_{2}=\{\zeta \in T:|\zeta-z| \leq \rho(z, E) / 2\}$ be the two possible parts of $\partial H(z)$. Then

$$
\left|\int_{\gamma_{1}} \frac{\bar{\partial} \phi(\zeta)}{h(\zeta)} \frac{d \bar{\zeta}}{\zeta-z}\right| \leq \text { const } \int_{\gamma_{1}} \frac{1}{\rho\left(\zeta, E_{0}\right)^{1+\alpha} \psi\left(\zeta, E_{1}\right)} \frac{|d \zeta|}{|\zeta-z|} .
$$

For $\zeta \in \gamma_{1}, \rho\left(\zeta, E_{0}\right) \sim \rho\left(z, E_{0}\right), \rho(\zeta, E) \sim \rho(z, E)$ and so $\psi\left(\zeta, E_{1}\right) \sim$ $\psi\left(z, E_{1}\right)$. So the integral over $\gamma_{1}$ is bounded by

$$
\text { const } \rho\left(z, E_{0}\right)^{-1-\alpha} \psi\left(z, E_{1}\right)^{-1} \text {. }
$$

At last, we can write the integral over $\gamma_{2}$ in the form

$$
\int_{\gamma_{2}} \frac{\bar{\partial} \phi(\zeta)}{\zeta^{2} h(\zeta)} \frac{d \zeta}{\zeta-z}
$$

To estimate it, we use Lemma 2.6 with $\varphi(\zeta)=\bar{\partial} \phi(\zeta) / \zeta^{2} h(\zeta)$ (recall that $\bar{\partial} \phi$ and $h$ are $C^{\infty}$ in $\bar{D} \backslash E$ ) and $n=0$. With the notations of that lemma, it is easily seen that $M_{0} \leq c \rho\left(z, E_{0}\right)^{-1-\alpha}$ and $M_{1} \leq c \rho\left(z, E_{0}\right)^{-2-\alpha}$. Since the length of $\gamma_{2}$ is less than const $\rho\left(z, E_{0}\right)$, we finally obtain the bound const $\rho\left(z, E_{0}\right)^{-1-\alpha}$.

Proof of the sufficiency continued. We now finish the proof in case $E_{1}$ is not a Carleson set. By Lemma 2.7, we can assume in this case that $E_{1}=\left(b_{n}\right)_{n} \cup\left(c_{n}\right)_{n}$ where $\left(b_{n}\right)$ and $\left(c_{n}\right)$ are Carleson sequences and $\psi\left(b_{n}, c_{n}\right) \rightarrow 0$. We put $F=E_{0} \cup\left\{\left(b_{n}\right)\right\}, G=E_{0} \cup\left\{\left(c_{n}\right)\right\}$. Then, $F, G$ are semi-Stolzian (CK)-sets and $F_{0}=G_{0}=E_{0}, E=F \cup G$.

Given $\phi \in \operatorname{Lip}_{1}(E)$, by Lemma 4.1, there exists $f_{1} \in \operatorname{Lip} 1$ such that $f_{1}=\phi$ on $F$. Hence

$$
\left|\phi_{1}(z)-f_{1}(z)\right| \leq \text { const } \rho(z, F), \quad z \in E .
$$


We look for the desired $f$ in the form $f=f_{1}+B_{F} F_{1} g$ with $g \in H(D)$, because in this manner $f=f_{1}=\phi$ on $F$. In order that $f=\phi$ on $\left(c_{n}\right)$ we need $g\left(c_{n}\right)=\lambda_{n}$ where $\lambda_{n}=\phi\left(c_{n}\right)-f_{1}\left(c_{n}\right) / B_{F}\left(c_{n}\right) F_{1}\left(c_{n}\right)$ if $c_{n} \notin F, \lambda_{n}=$ 0 if $c_{n} \in F$. To assure that $f \in \operatorname{Lip} 1$ we must impose that $B_{F} F_{1} g$ has a bounded derivative. This is true if $g$ is bounded and $\left|g^{\prime}(z)\right|=$ $O\left(\rho\left(z, F_{0}\right)^{-1}\right)$. The relation (10) implies that $\left(\lambda_{n}\right)$ is a bounded sequence. Since $G_{0}=F_{0}$, the proof of Theorem 1 will thus be finished as soon as we prove the following $H^{\infty}$-interpolation result for (semi-Stolzian) (CK)-sets with control on the derivative.

LEMMA 4.2. Let $G$ be a semi-Stolzian (CK)-set, $G_{1}=\left(c_{n}\right)$ and let $\left(\lambda_{n}\right)$ be a bounded sequence. Then there exists $g \in H^{\infty}$ such that $g\left(c_{n}\right)=\lambda_{n}$ and $\left|g^{\prime}(z)\right|=O\left(\rho\left(z, G_{0}\right)^{-1}\right)$.

Proof of Lemma 4.2. Let $\delta>0$ be such that the discs $D_{n}=$ $D\left(c_{n}, \delta\left(1-\left|c_{n}\right|\right)\right)$ are disjoint. We define

$$
\phi(z)=\sum_{n} \lambda_{n} \chi\left(\frac{2\left|z-c_{n}\right|^{2}}{\delta^{2}\left(1-\left|c_{n}\right|\right)^{2}}\right),
$$

where $\chi \in C^{\infty}\left(R^{+}\right)$is such that $\chi(t)=1, t \in[0,1 / 4], \chi(t)=0, t \in$ $[1 / 2, \infty), 0 \leq \chi \leq 1$. $\phi$ is a bounded $C^{\infty}$ function in $D, \phi\left(c_{n}\right)=\lambda_{n}$ and

$$
|\bar{\partial} \phi(z)| \leq\left|\lambda_{n}\right|\left\|\chi^{\prime}\right\|_{\infty} \frac{2\left|z-c_{n}\right|}{\delta^{2}\left(1-\left|c_{n}\right|\right)^{2}}, \quad z \in D_{n} .
$$

We define $g=P\left(\phi, F_{\alpha} B_{G}\right)$ where $\alpha$ is as in Lemmas 2.4 and 2.5 (for $G$ ). In this case

$$
|v(z)| \leq \mathrm{const} \sum_{n} \int_{\left|\zeta-c_{n}\right| \leq \delta\left(1-\left|c_{n}\right|\right) / 2} \frac{|\bar{\partial} \phi(\zeta)|}{\rho\left(\zeta, G_{0}\right)^{\alpha} \psi\left(\zeta, G_{1}\right)} \frac{d m(\zeta)}{|\zeta-z|}, \quad z \notin G
$$

Hence

$$
|v(z)| \leq \operatorname{const} \sum_{n}\left(1-\left|c_{n}\right|\right)^{-1-\alpha} \int_{\left|\zeta-c_{n}\right| \leq \delta\left(1-\left|c_{n}\right|\right) / 2} \frac{d m(\zeta)}{|\zeta-z|}
$$

An easy computation shows that the last integral is bounded by const $\left(1-\left|c_{n}\right|^{2}\right) /\left|z-c_{n}\right|$ if $z \notin D_{n}$ and by const $\left(1-\left|c_{n}\right|\right)$ if $z \in D_{n}$. Then (4) and (5) lead to

$$
|v(z)|=O\left(\rho\left(z, G_{0}\right)^{-\alpha}\right) .
$$


This gives $\left|v(z) F_{\alpha}(z) B_{G}(z)\right| \leq$ const $\psi\left(z, G_{1}\right)$ and so $g \in H^{\infty}, g\left(c_{n}\right)=$ $\phi\left(c_{n}\right)=\lambda_{n}$. Now it remains to prove the estimate for $g^{\prime}$. Since $\rho\left(z, G_{0}\right) \sim$ $1-\left|c_{n}\right|$ in $D_{n}$ it is enough to prove it for $z \notin \cup_{n} D_{n}$. In this case

$$
\partial v(z)=-\frac{1}{\pi} \int_{D} \frac{\bar{\partial} \phi(\zeta)}{F_{\alpha}(\zeta) B_{G}(\zeta)} \frac{d m(\zeta)}{(\zeta-z)^{2}}
$$

and, just as before

$$
\begin{aligned}
|\partial v(z)| & \leq \mathrm{const} \sum_{n}\left(1-\left|c_{n}\right|\right)^{-1-\alpha} \int_{\left|\zeta-c_{n}\right| \leq \delta\left(1-\left|c_{n}\right|\right) / 2} \frac{d m(\zeta)}{|\zeta-z|^{2}} \\
& \leq \operatorname{const} \sum_{n} \frac{\left(1-\left|c_{n}\right|\right)^{1-\alpha}}{\left|z-c_{n}\right|^{2}} \leq \operatorname{const} \rho(z, G)^{-1} \rho\left(z, G_{0}\right)^{-\alpha} \\
& =\operatorname{const} \rho\left(z, G_{0}\right)^{-1-\alpha}, \quad z \notin \bigcup_{n} D_{n} .
\end{aligned}
$$

From this and the usual estimates on $B_{G}$ and $F_{\alpha}$ we find that $\left|g^{\prime}(z)\right|=$ $O\left(\rho\left(z, G_{0}\right)^{-1}\right)$, as required.

This ends the proof of Theorem 1.

REMARK 4.3. The method of proof of Lemma 4.1 also works in the Lip $s$-case, $0<s<1$, thus obtaining another proof of Dyn'kin's theorem. The estimates for $\phi$ would be changed to

$$
\begin{gathered}
|\bar{\partial} \phi(\zeta)| \leq \text { const } \rho(\zeta, E)^{s-1}, \quad|\partial \phi(\zeta)| \leq \text { const } \rho(\zeta, E)^{s-1} \\
|\partial \bar{\partial} \phi(\zeta)| \leq \text { const } \rho(\zeta, E)^{s-2}
\end{gathered}
$$

for $\zeta \notin E$. With $h=B_{E} F_{s+\alpha}$, the corresponding $v$ in the standard method of interpolation would satisfy

$$
\begin{aligned}
|v(z)| & =O\left(\rho\left(z, E_{0}\right)^{-\alpha} \psi\left(z, E_{1}\right)^{s-1}\right), \quad z \in \bar{D} \backslash E \\
|\partial v(z)| & =O\left(\rho\left(z, E_{0}\right)^{-\alpha-1} \psi\left(z, E_{1}\right)^{s-2}\right), \quad z \in \bar{D} \backslash E .
\end{aligned}
$$

This is proved in the same manner using the case $s<1$ in Lemma 2.5. With $f=P(\phi, h)$, we obtain

$$
\left|f^{\prime}(z)\right| \leq \text { const } \rho(z, E)^{s-1}, \quad z \in \bar{D} \backslash E .
$$

If $z \notin \cup_{n} D_{n}$ this implies $\left|f^{\prime}(z)\right| \leq \operatorname{const}(1-|z|)^{s-1}$. Since $1-|z| \sim 1$ - $\left|a_{n}\right|$ for $z \in D_{n}$, we conclude that $\left|f^{\prime}(z)\right| \leq \operatorname{const}(1-|z|)^{s-1}$ for all $z$. By the well-known Hardy-Littlewood theorem (see [3], pg. 74) $f$ is in Lip $s$. 
But now

$$
|h(z) v(z)|=O\left(\rho\left(z, E_{0}\right)^{s} \psi\left(z, E_{1}\right)^{s}\right), \quad z \in \bar{D} \backslash E,
$$

so that $f=\phi-h v$ interpolates $\phi$ on $E$.

V. Proof of Theorems 2 and 3. The estimates in the proof of Theorem 2 involve a non-decreasing, concave, continuous function $\omega$ : $[0, \infty) \rightarrow R$ with $\omega(0)=0$. We shall call such a function a modulus of continuity.

We begin with the analogues of Lemmas 2.4 and 2.5:

LEMMA 5.1. Let $E$ be a semi-Stolzian (CK)-set and let $\alpha$ be as in Lemmas 2.3, 2.4 and 2.5. Then

(a) If $\left|\mu_{n}\right|=o(1)$ as $1-\left|a_{n}\right| \rightarrow 0$, then

$$
\begin{aligned}
& \sum_{n}\left|\mu_{n}\right| \frac{\left(1-\left|a_{n}\right|\right)^{1-\alpha}}{\left|z-a_{n}\right|}=o\left(\rho\left(z, E_{0}\right)^{-\alpha}\right), \quad z \notin \bigcup_{n} D_{n}, \\
& \sum_{m \neq n}\left|\mu_{m}\right| \frac{\left(1-\left|a_{m}\right|\right)^{1-\alpha}}{\left|z-a_{m}\right|}=o\left(\rho\left(z, E_{0}\right)^{-\alpha}\right), \quad z \in D_{n} .
\end{aligned}
$$

(b) If $\omega$ is a modulus of continuity,

$$
\begin{aligned}
& \int \frac{\omega(\rho(\zeta, E))}{D \rho\left(\zeta, E_{0}\right)^{1+\alpha} \psi\left(\zeta, E_{1}\right)} \frac{d m(\zeta)}{|\zeta-z|} \\
&=o\left(\rho\left(z, E_{0}\right)^{-\alpha}\left(\text { const }+\left|\log \psi\left(z, E_{1}\right)\right|\right)\right)
\end{aligned}
$$

as $\rho\left(z, E_{0}\right) \rightarrow 0, z \in \bar{D} \backslash E$.

Proof. (b) Break the integral into two parts corresponding to $\{\zeta$ : $\left.\rho(\zeta, E) \leq \rho\left(z, E_{0}\right)^{\alpha / 2(2+\alpha)}\right\}$ (near $\left.E\right)$ and the complementary. In the first region, $\omega(\rho(\zeta, E)) \leq$ const $\omega\left(\rho\left(z, E_{0}\right)^{\alpha / 2(2+\alpha)}\right)$ and by (6) its contribution satisfies (13). In the second region, $\rho\left(\zeta, E_{0}\right) \geq \rho\left(z, E_{0}\right)^{\alpha / 2(2+\alpha)}$ and $\psi\left(\zeta, E_{1}\right) \leq$ const $\rho\left(z, E_{0}\right)^{\alpha / 2(2+\alpha)}$ and hence the integral over it is bounded by

$$
\rho\left(z, E_{0}\right)^{-\alpha / 2} \int_{D} \frac{d m(\zeta)}{|\zeta-z|}=\text { const } \rho\left(z, E_{0}\right)^{-\alpha / 2}
$$

which is of type (13).

The proof of (a) follows the same pattern but using (4) and (5) instead of (6). 
Proof of the necessity. We just verify that (C) holds, the condition (K) being then obtained as in the Lip 1-case. For $n$ fixed, let's consider the jet $\phi_{n}=\left(\phi_{n}, \phi_{n z}, 0\right)$ where $\phi_{n} \equiv 0$ and $\phi_{n z}=0$ on $E$ except on $a_{n}$ where $\phi_{n z}\left(a_{n}\right)=1$ (that is, $\phi_{n}$ is the induced jet by some function which equals $z-a_{n}$ in a neighbourhood of $a_{n}$ and zero in a neighbourhood of $E \backslash a_{n}$ ). The norm of $\phi_{n}$ in $C^{1}(E)$ is 1 . By the open mapping theorem, there exists $f_{n} \in A^{1}$ interpolating $\phi_{n}$ and its derivative on $E$ and such that $\left\|f_{n}\right\|_{A^{1}} \leq$ const. Then $g_{n} \equiv f_{n}^{\prime}$ are uniformly bounded functions such that $g_{n}\left(a_{m}\right)=$ $\delta_{m n}$. From this point on, the classical argument for $H^{\infty}$ applies to show that $(\mathrm{C})$ holds.

Proof of the sufficiency. Given $\phi \in C^{1}(\mathbf{C})$ with $\partial \phi / \partial \bar{z}=0$ on a (CK)-set $E$ we define a new jet $\tilde{\phi}$ on the semi-Stolzian (CK)-set given by Lemma 2.1 by putting $\tilde{\phi}=\phi$ and $\tilde{\phi}_{z}=(\partial \phi / \partial z)-\bar{z}^{2} \partial \phi / \partial \bar{z}, \tilde{\phi}_{\bar{z}}=0$ on $\tilde{E}$. It is easily seen that $\tilde{\phi}$ is a Whitney jet on $\tilde{E}$ which extends the jet $\phi$ on $E$. Hence we can also assume that $E$ is a semi-Stolzian (CK)-set.

As in Theorem 1, the proof is obtained in two steps. The first one is similar to Lemma 4.1.

LEMMA 5.2. Let $E$ be a semi-Stolzian (CK)-set and let $\phi \in C^{1}(E)$ be such that $\phi_{\bar{z}}=0$. Then there exists $f_{1} \in A^{1}$ such that $f_{1}=\phi$ on $E$ and $f_{1}^{\prime}=\phi_{z}$ on $E_{0}$.

Proof. By Whitney's extension theorem ([7]) we can suppose here that $\phi \in C^{\infty}(\mathbf{C} \backslash E)$ and that

$$
\begin{gathered}
|\bar{\partial} \phi(\zeta)| \leq \text { const } \omega(\rho(\zeta, E)), \\
|\partial \bar{\partial} \phi(\zeta)| \leq \text { const } \omega(\rho(\zeta, E)) \rho(\zeta, E)^{-1}, \quad \zeta \notin E .
\end{gathered}
$$

Let $h=B_{E} F_{1+\alpha}$ and let $f_{1}=P(\phi, h)$, so that $f_{1}$ and $f_{1}^{\prime}$ have continuous extensions to $T \backslash E$. We claim that

$$
|v(z)|=o\left(\rho\left(z, E_{0}\right)^{-\alpha}\left(\text { const }+\left|\log \psi\left(z, E_{1}\right)\right|\right)\right)
$$

$$
|\partial v(z)|=o\left(\rho\left(z, E_{0}\right)^{-\alpha-1} \psi\left(z, E_{1}\right)^{-1}\left(\text { const }+\left|\log \psi\left(z, E_{1}\right)\right|\right)\right)
$$

as $\rho\left(z, E_{0}\right) \rightarrow 0, z \notin E$ (recall that $v$ and $\partial v$ are continuous functions on $\bar{D} \backslash E)$. From these we see that

$$
\begin{gathered}
|(v h)(z)|=o\left(\rho\left(z, E_{0}\right) \psi\left(z, E_{1}\right)\left(\text { const }+\left|\log \psi\left(z, E_{1}\right)\right|\right)\right) \\
\text { as } \rho\left(z, E_{0}\right) \rightarrow 0, z \notin E, \\
|\partial(v h)(z)|=o(1) \quad \text { as } \rho\left(z, E_{0}\right) \rightarrow 0, z \in T \backslash E_{0} .
\end{gathered}
$$


The growth estimate for $v h$ implies that $f_{1}$ interpolates $\phi$ on $E$ and $f_{1} \in A$. The estimate for $\partial(v h)$ proves that $f_{1}^{\prime}(z)$ has a limit as $z \rightarrow a \in E_{0}$, with $z \in T \backslash E_{0}$. This limit equals $\partial \phi(a)$. This implies that $F(t)=f_{1}\left(e^{i t}\right)$ is a differentiable function of $t$, and hence $f_{1} \in A^{1}$. So it only remains to prove (14) and (15) (in fact (15) is just needed for $z \in T \backslash E_{0}$ ). We have

$$
|v(z)| \leq \mathrm{const} \int_{D} \frac{\omega(\rho(\zeta, E))}{\rho\left(\zeta, E_{0}\right)^{1+\alpha} \psi\left(\zeta, E_{1}\right)} \frac{d m(\zeta)}{|\zeta-z|}
$$

and so (14) follows from (13) in Lemma 5.1. For $\partial v(z)$ we have as in Lemma 4.1

$$
\begin{aligned}
|\partial v(z)| \leq & \text { const } \int_{D \backslash H(z)} \frac{\omega(\rho(\zeta, E))}{\rho\left(\zeta, E_{0}\right)^{1+\alpha} \psi\left(\zeta, E_{1}\right)} \frac{d m(\zeta)}{|\zeta-z|^{2}} \\
& + \text { const } \int_{H(z)} \frac{\omega(\rho(\zeta, E)) \rho(\zeta, E)^{-1}}{\rho\left(\zeta, E_{0}\right)^{1+\alpha} \psi\left(\zeta, E_{1}\right)} \frac{d m(\zeta)}{|\zeta-z|} \\
& + \text { const }\left|\int_{\partial H(z)} \frac{\bar{\partial} \phi(\zeta)}{h(\zeta)} \frac{d \bar{\zeta}}{\zeta-z}\right|
\end{aligned}
$$

Since $|\zeta-z| \geq \rho(z, E) / 2$ for $\zeta \notin H(z)$, the first integral on the right is bounded by $\rho(z, E)^{-1}$ times the integral in (13), and so it satisfies the estimate. For $\zeta \in H(z)$ one has $\omega(\rho(\zeta, E)) \leq$ const $\omega(\rho(z, E))$. Therefore the integral over $H(z)$ is bounded by $\omega(\rho(z, E))$ times the integral in (7) with $s=1$ and the estimate follows again. Finally, the integral over $\partial H(z)$ is estimated as in Lemma 4.1.

Proof of the sufficiency continued. Let $f_{1}$ be the function obtained in Lemma 5.2. Let $\mu_{n}=\phi_{z}\left(a_{n}\right)-f_{1}^{\prime}\left(a_{n}\right)$. Since $f_{1}^{\prime}$ interpolates $\phi_{z}$ on $E_{0}$, $\left|\mu_{n}\right|=o(1)$ as $1-\left|a_{n}\right| \rightarrow 0$. To finish the proof of the theorem we must look for a $g \in A^{1}$ vanishing on $E$ and such that $g^{\prime}=0$ on $E_{0}, g^{\prime}\left(a_{n}\right)=\mu_{n}$ and then put $f=f_{1}+g$. We define

$$
\Gamma(z)=\sum_{n} \mu_{n}\left(z-a_{n}\right) \chi\left(\frac{2\left|z-a_{n}\right|^{2}}{\delta^{2}\left(1-\left|a_{n}\right|\right)^{2}}\right)
$$

where $\chi$ is as in the proof of Lemma 4.2. Clearly, for $z \in D_{n}$ $|\Gamma(z)| \leq\left|\mu_{n}\right|\left|z-a_{n}\right|, \quad|\partial \Gamma(z)| \leq \mathrm{const}\left|\mu_{n}\right|, \quad|\bar{\partial} \Gamma(z)| \leq \mathrm{const}\left|\mu_{n}\right| \psi\left(z, a_{n}\right)^{2}$. Hence, $\Gamma, \partial \Gamma$ and $\bar{\partial} \Gamma$ are $o(1)$ as $|z| \rightarrow 1$. Therefore $\Gamma$ is a $C^{1}$ function in $\bar{D}$ which is flat on $T, C^{\infty}$ outside $E$. 
With $h=F_{1+\alpha} B_{E}^{2}$ we define $g=P(\Gamma, h)$. By the comments in $\S I I I$ and $g^{\prime}$ are holomorphic functions, continuous in $\bar{D} \backslash E$. In this case

$$
|v(z)| \leq \mathrm{const} \sum_{n}\left|\mu_{n}\right|\left(1-\left|a_{n}\right|\right)^{-1-\alpha} \int_{\left|\zeta-a_{n}\right| \leq \delta\left(1-\left|a_{n}\right|\right) / 2} \frac{d m(\zeta)}{|\zeta-z|} .
$$

The last integral is bounded by const $\left(1-\left|a_{n}\right|\right)^{2} /\left|z-a_{n}\right|$ if $z \notin D_{n}$ and by const $\left(1-\left|a_{n}\right|\right)$ if $z \in D_{n}$. Then (a) of Lemma 5.1 gives

$$
|v(z)|=o\left(\rho\left(z, E_{0}\right)^{-\alpha}\right), \quad z \notin E \text {, as } \rho\left(z, E_{0}\right) \rightarrow 0 .
$$

This implies $|h(z) v(z)|=o\left(\rho\left(z, E_{0}\right) \psi\left(z, E_{1}\right)^{2}\right)$ as $\rho\left(z, E_{0}\right) \rightarrow 0$ and so $g \in A, g$ interpolates $\Gamma$ on $E$ (and hence $g=0$ on $E$ ) and $g^{\prime}\left(a_{n}\right)=$ $\partial \Gamma\left(a_{n}\right)=\mu_{n}$. Therefore, all we must verify is that $g \in A^{1}$ and $g^{\prime}=0$ on $E_{0}$. As before, to see this it is enough to see that $\lim _{z \rightarrow E_{0} ; z \in T} g^{\prime}(z)=0$. Since $\partial \Gamma=0$ on $E_{0}$ this will follow from an estimate of type

$$
|\partial(v h)(z)|=o(1) \quad \text { as } \rho\left(z, E_{0}\right) \rightarrow 0, z \in T \backslash E_{0}
$$

which is turn will follow from

$$
|\partial v(z)|=o\left(\rho\left(z, E_{0}\right)^{-\alpha-1}\right), \quad z \in D \backslash \bigcup_{n} D_{n} .
$$

For these $z$,

$$
|\partial v(z)| \leq \operatorname{const} \sum_{n} \frac{\left|\mu_{n}\right|\left(1-\left|a_{n}\right|\right)^{1-\alpha}}{\left|z-a_{n}\right|^{2}} \leq c \rho\left(z, E_{0}\right)^{-1} \sum_{n}\left|\mu_{n}\right| \frac{\left(1-\left|a_{n}\right|\right)^{1-\alpha}}{\left|z-a_{n}\right|}
$$

and (16) follows from (a) in Lemma 5.1. Thus the proof of Theorem 2 is completely finished.

Sketch of the proof of Theorem 3. The necessity of condition (C) is obvious. We indicate the proof of the sufficiency. Given $\phi \in \operatorname{Lip}_{1}(E)$ and $\left(\lambda_{n}\right) \in l^{\infty}$ ( $E$ semi-Stolzian), let $f_{1}$ be as in Lemma 4.1 and define $\Gamma$ as above, with $\mu_{n}=\lambda_{n}-f_{1}^{\prime}\left(a_{n}\right)$, which in this case is a bounded sequence. $\Gamma$ is continuous on $\bar{D}, C^{\infty}$ in $\bar{D} \backslash E$ and vanishes on $E$. Also,

$$
|\bar{\partial} \Gamma(z)| \leq \text { const } \psi\left(z, a_{n}\right)^{2}, \quad z \in D_{n} .
$$

As above, with $h=F_{1+\alpha} B_{E}^{2}$ put $g=P(\Gamma, h)$. The corresponding $v$ satisfies

$$
\begin{aligned}
|v(z)| & =O\left(\rho\left(z, E_{0}\right)^{-\alpha}\right), \quad z \notin E, \\
|\partial v(z)| & =O\left(\rho\left(z, E_{0}\right)^{-\alpha-1}\right), \quad z \in D \backslash \bigcup_{n} D_{n} .
\end{aligned}
$$


From these we derive the relations

$$
\begin{aligned}
& |h(z) v(z)|=O\left(\rho\left(z, E_{0}\right) \psi\left(z, E_{1}\right)^{2}\right), \quad z \notin E, \\
& |\partial(v h)(z)|=O(1), \quad z \in D \backslash \bigcup_{n} D_{n} .
\end{aligned}
$$

The second implies that $g^{\prime}=\partial \Gamma-\partial(v h)$ is bounded in $D \backslash \cup_{n} D_{n}$, hence bounded in $D$ and so $g \in \operatorname{Lip} 1$. The first shows that $v h$ vanishes once in $E_{0}$ and twice at each point in $E_{1}$ so that $g=\Gamma=0$ on $E$ and $g^{\prime}\left(a_{n}\right)=$ $\partial \Gamma\left(a_{n}\right)=\mu_{n}$. Then $f=f_{1}+g$ performs the desired interpolation.

\section{REFERENCES}

1. J. Bruna, Boundary interpolations sets for holomorphic functions smooth to the boundary and BMO, Trans. Amer. Math. Soc., 264, 2 (1981), 391-409.

2. L. Carleson, Sets of uniqueness for functions regular in the unit circle, Acta Math., 87 (1952), 325-345.

3. P. L. Duren, Theory of $H^{p}$ Spaces, Academic Press, New York, 1970.

4. E. M. Dyn'kin, Free interpolation sets for Hölder classes, Mat. Sb., 109 (151) (1979), 107-128 = Math. USSR-Sb., 37 (1980), 97-117.

5. A. M. Kotocigov, Interpolation by analytic functions that are smooth up to the boundary, Zap. Naucn. Sem. Leningrad. Otdel. Mat. Inst. Steklov. (LOMI), 30 (1972), 167-169; English transl. in J. Soviet Math., 4 (1975), no. 4.

6. E. P. Kronstadt, Interpolating sequences for functions satisfying a Lipschitz condition, Pacific J. Math., 63, 1 (1976).

7. B. Malgrange, Ideals of Differentiable Functions, Tata Institute, Bombay, 1966.

8. E. M. Stein, Singular integrals and differentiability properties of functions, Princeton Univ. Press, Princeton, N. J., 1970.

9. V. I. Vasjunin, Bases of eigensubspaces and nonclassical interpolation problems, Funkcional. Anal. i Prilozen. 9 (1975), no. 4, 65-66; English transl. in Functional Anal. Appl., 9 (1975).

10. S. A. Vinogradov and V. P. Havin, Free interpolation in $H^{\infty}$ and in certain other classes of functions. I, II, Zap. Naucn. Sem. Leningrad Otdel. Mat. Inst. Steklov. (LOMI), 47 (1974), 15-54; 56 (1976), 12-58; English transl. in J. Soviet. Math., 9 (1978), no. 2.

Received December 11, 1981 and in revised form April 1, 1982.

Universitat Autonoma De Barcelona

Seccio De Mathematiques

Bellaterra (BARCElona) SPAin 



\section{PACIFIC JOURNAL OF MATHEMATICS EDITORS}

Donald BABBITT (Managing Editor)

University of California

Los Angeles, CA 90024

Hugo Rossi

University of Utah

Salt Lake City, UT 84112

C. C. Moore and Arthur Ogus

University of California

Berkeley, CA 94720
J. DugundiI

Department of Mathematics

University of Southern California

Los Angeles, CA 90089-1113

R. Finn and H. SAMELSON

Stanford University

Stanford, CA 94305

ASSOCIATE EDITORS
R. ARENS
E. F. BECKENBACH
B. H. NeUmanN
F. WOLF
K. YosHIDA (1906-1982)

\section{SUPPORTING INSTITUTIONS}

UNIVERSITY OF ARIZONA

UNIVERSITY OF BRITISH COLUMBIA

CALIFORNIA INSTITUTE OF TECHNOLOGY

UNIVERSITY OF CALIFORNIA

MONTANA STATE UNIVERSITY

UNIVERSITY OF NEVADA, RENO

NEW MEXICO STATE UNIVERSITY

OREGON STATE UNIVERSITY
UNIVERSITY OF OREGON

UNIVERSITY OF SOUTHERN CALIFORNIA

STANFORD UNIVERSITY

UNIVERSITY OF HAWAII

UNIVERSITY OF TOKYO

UNIVERSITY OF UTAH

WASHINGTON STATE UNIVERSITY

UNIVERSITY OF WASHINGTON 


\section{Pacific Journal of Mathematics}

\section{Vol. 108, No. $1 \quad$ March, 1983}

Waleed A. Al-Salam and A. Verma, $q$-Konhauser polynomials $\ldots \ldots \ldots \ldots 1$

Alfred David Andrew, The Banach space JT is primary $\ldots \ldots \ldots \ldots \ldots . . .6$

Thomas E. Bengtson, Bessel functions on $P_{n} \ldots \ldots \ldots \ldots \ldots \ldots$

Joaquim Bruna Floris and Francesc Tugores, Free interpolation for

holomorphic functions regular to the boundary $\ldots \ldots \ldots \ldots \ldots \ldots \ldots \ldots$

Peter Dierolf and Susanne Dierolf, Topological properties of the dual pair

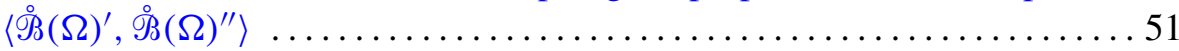

Gerald Arthur Edgar, An ordering for the Banach spaces $\ldots \ldots \ldots \ldots \ldots 83$

Basil Gordon, A proof of the Bender-Knuth conjecture . . . . . . . . . . . . . 99

Harold T. Hodes, A minimal upper bound on a sequence of Turing degrees

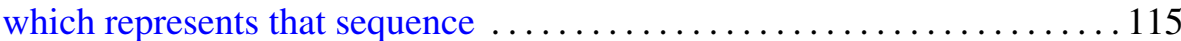

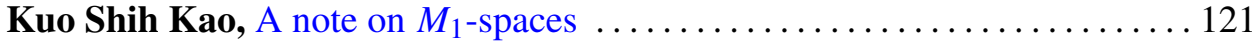

Frank Kost, Topological extensions of product spaces ................ 129

Eva Lowen-Colebunders, On the convergence of closed and compact

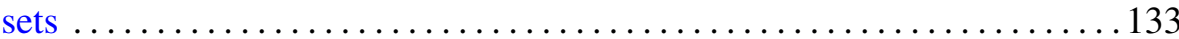

Doron Lubinsky, Divergence of complex rational approximations . . . . . . 141

Warren May and Elias Hanna Toubassi, Endomorphisms of rank one

mixed modules over discrete valuation rings $\ldots \ldots \ldots \ldots \ldots \ldots \ldots \ldots \ldots$

Richard Patrick Morton, The quadratic number fields with cyclic

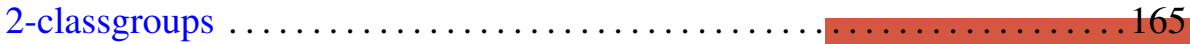

Roderic Murufas, Rank of positive matrix measures . . . . . . . . . . . . 177

Helga Schirmer, Fixed point sets of homotopies . . . . . . . . . . . . . 191

E. Taflin, Analytic linearization of the Korteweg-de Vries equation ........ 203

James Thomas Vance, Jr., $L^{p}$-boundedness of the multiple Hilbert

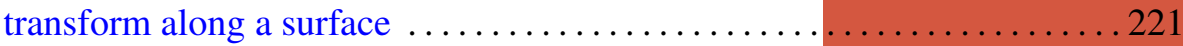

Hiroshi Yamaguchi, A property of some Fourier-Stieltjes transforms . . . . . 243 